\title{
Prevalence of Central Nervous System Polypharmacy and Associations with Overdose and Suicide-Related Behaviors in Iraq and Afghanistan War Veterans in VA Care 2010-2011
}

\author{
Garen A. Collett ${ }^{2} \cdot$ Kangwon Song $^{1,2,3} \cdot$ Carlos A. Jaramillo $^{1,2} \cdot$ Jennifer S. Potter $^{2}$. \\ Erin P. Finley ${ }^{1,2} \cdot$ Mary Jo Pugh ${ }^{1,2}$
}

Published online: 8 January 2016

(c) The Author(s) 2016. This article is published with open access at Springerlink.com

\begin{abstract}
Background The increase in the quantities of central nervous system (CNS)-acting medications prescribed has coincided with increases in overdose mortality, suiciderelated behaviors, and unintentional deaths in military personnel deployed in support of the wars in Iraq and Afghanistan. Data on the extent and impact of prescribing multiple CNS drugs among Iraq and Afghanistan Veterans (IAVs) are sparse.
\end{abstract}

Electronic supplementary material The online version of this article (doi:10.1007/s40801-015-0055-0) contains supplementary material, which is available to authorized users.

Mary Jo Pugh

pughm@uthscsa.edu

Garen A. Collett

garenc@gmail.com

Kangwon Song

Songk@uthscsa.edu

Carlos A. Jaramillo

jaramilloc3@uthscsa.edu

Jennifer S. Potter

potterjs@uthscsa.edu

Erin P. Finley

finleye@uthscsa.edu

1 Research, and Polytrauma Rehabilitation Center, South Texas Veterans Healthcare System, 7400 Merton Minter BLVD, San Antonio, TX, USA

2 Departments of Epidemiology and Biostatistics, Medicine, Psychiatry, Anesthesiology, and Rehabilitation Medicine, University of Texas Health Science Center San Antonio, 7703 Floyd Curl Drive, San Antonio, TX 78229, USA

3 College of Pharmacy, University of Texas, Austin, TX, USA
Objectives We sought to identify the characteristics of IAVs with CNS polypharmacy and examine the association of CNS polypharmacy with drug overdose and suiciderelated behaviors controlling for known risk factors.

Methods This cross-sectional cohort study examined national data of Iraq and Afghanistan Veterans $(N=311,400)$ who used the Veterans Health Administration (VHA) during the fiscal year 2011. CNS polypharmacy was defined as five or more CNS-acting medications; drug/alcohol overdose and suicide-related behaviors were identified using ICD-9-CM codes. Demographic and clinical characteristics associated with CNS polypharmacy were identified using a multivariable logistic regression model.

Results We found that 25,546 (8.4\%) of Iraq and Afghanistan Veterans had CNS polypharmacy. Those with only post-traumatic stress disorder (PTSD) (adjusted odds ratio (AOR) 6.50, $99 \%$ confidence interval (CI) 5.96-7.10), only depression (AOR 6.42, $99 \%$ CI 5.86-7.04), co-morbid PTSD and depression (AOR 12.98, $99 \%$ CI 11.97-14.07), and co-morbid traumatic brain injury (TBI), PTSD, and depression (AOR 15.30, $99 \% \mathrm{CI}$ 14.00-16.73) had the highest odds of CNS polypharmacy. After controlling for these co-morbid conditions, CNS polypharmacy was significantly associated with drug/alcohol overdose and suicide-related behavior.

Conclusion CNS polypharmacy was most strongly associated with PTSD, depression, and TBI, and independently associated with overdose and suicide-related behavior after controlling for known risk factors. These findings suggest that CNS polypharmacy may be used as an indicator of risk for adverse outcomes. Further research should evaluate whether CNS polypharmacy may be used as a trigger for evaluation of the current care provided to these individuals. 


\section{Key Points}

Over $8 \%$ of Iraq and Afghanistan Veterans received five or more CNS-acting medications in 2011.

Multimorbidity of mental disorders and traumatic brain injury was strongly associated with CNS polypharmacy.

Suicide-related behavior and drug/alcohol overdose were significantly associated with CNS polypharmacy.

\section{Introduction}

Recent Department of Defense (DoD) reports on medication use among active duty service members (ADSMs) deployed in support of conflicts in Iraq and Afghanistan indicate potentially problematic use of central nervous system (CNS)-acting drugs. Jeffery and colleagues found that nearly a third of all ADSM received one or more prescriptions for opioids, CNS depressants, or stimulants during the fiscal year 2010 [1]. According to the Army Institute of Public Health, $46 \%$ of those who completed suicides, $90 \%$ of those who attempted suicide, and $87 \%$ of those with suicidal ideation received a CNS-acting prescription the year preceding the event [2].

Reports of unintentional overdose by ADSMs on multiple CNS medication (CNS polypharmacy) led to significant concern that CNS medication and CNS polypharmacy may be related to unintentional overdose which appears to be suicide in ADSMs and Veterans [3, 4] - a phenomenon that is parallel to the high prevalence of unintentional overdose in the broader US population [5]. Since 1999, unintentional injury deaths from poisoning have increased almost $250 \%$, surpassing motor vehicle accidents as the number one cause of death from unintentional injury in the USA [5]. There is a high prevalence of pain, mental health conditions, and traumatic brain injury (TBI) among ADSMs and Veterans who served in support of the wars in Iraq and Afghanistan [6,7]. These conditions are frequently co-morbid and are typically treated with CNS medications; however, the CNS drug burden in this population is poorly understood.

Prior studies in the VA population have tended to focus on individual classes of CNS drugs [8, 9]. However, CNS polypharmacy among VA patients is not uncommon. Bernardy and colleagues [10] found that approximately $10 \%$ of all Veterans with PTSD received medications from three or more classes of medications that induce sedation in 2004, a rate that increased to $12 \%$ by 2011 . Despite this important work, the total burden of CNS medication usage among Veterans deployed in support of the Iraq/Afghanistan wars has not been systematically described. CNS polypharmacy is associated with an increased risk for drug/ alcohol overdose and suicide-related behaviors (SRBs); however, most studies have been conducted in populations with mental health co-morbidity, which complicates the interpretation of adverse outcomes $[3,11]$. Whether CNS polypharmacy is an independent risk factor for overdose or SRB after accounting for mental health co-morbidity is unknown.

This study examined the prevalence of CNS polypharmacy, demographic and clinical characteristics (co-morbid conditions) associated with CNS polypharmacy, and the association between CNS polypharmacy and drug/alcohol overdose and suicide-related behaviors (SRBs) in a national cohort of Iraq and Afghanistan Veterans (IAVs). We hypothesized that pain, mental health conditions, and TBI would be associated with CNS polypharmacy, and that CNS polypharmacy would be independently associated with overdose and SRB after controlling for known risk factors such as mental health co-morbidity.

\section{Methods}

\subsection{Participants}

Using the roster of Veterans who served in support of Post 9-11 conflicts (OEF/OIF/OND Roster) [12] we identified a cohort of individuals who received VA care in the fiscal year 2010-2011 (FY10-11; 1 October 2009-30 September 2011) as part of a larger study examining co-morbidity patterns in IAVs between 2001 and 2011. We included only those who received care at least once each year in FY10 and FY11. For those individuals who met inclusion criteria, we obtained all VA inpatient and outpatient administrative data from the national VA data repository in Austin, TX, and pharmacy data from the VA Pharmacy Benefits Management Strategic Health Group for FY10 and FY11. These claims files were merged using an encrypted identifier that is common for each individual in all datasets.

\subsection{Conceptual Model}

We used a traditional health-outcomes model that posits that health care utilization (CNS polypharmacy) is associated with predisposing, enabling, and need factors, and that outcomes such as overdose and suicide-related behavior are a function of baseline characteristics, patient clini$\mathrm{cal} /$ sociodemographic characteristics, treatment received, and the setting of care [13]. We hypothesized a priori that mental health co-morbidity, pain, and TBI would be 
associated with CNS polypharmacy and that, after controlling for characteristics associated with overdose and SRB in prior studies (age, sex, race, poverty, mental health co-morbidity, prior overdose, prior SRB [14, 15]), CNS polypharmacy would be independently associated with overdose and SRB.

\subsection{Measures}

\subsubsection{CNS Polypharmacy}

After compiling pharmacy data from the PBM database, we counted the number of unique CNS medications dispensed from VA pharmacies during FY11. Online Resource 1 provides the included drug classes and their specific medications. We excluded acetaminophen, aspirin, and choline salicylate/magnesium salicylate as these non-opioid analgesics do not act directly upon the CNS. Prior geriatric studies identified CNS polypharmacy as two or more CNSacting medications [16]. However, given multimorbidity in this population and clinical practice guideline recommendations for common co-morbidities (traumatic brain injury, post-traumatic stress disorder, depression, and pain) [1720], we used a more conservative definition-five or more CNS acting medications-for this young population with complex co-morbidity $[14,21]$.

\subsubsection{Demographic Characteristics}

We obtained age, sex, race/ethnicity, and branch of service using the OEF/OIF/OND roster [12]. We used VA administrative data to supplement missing demographics and to identify individuals with waived copayments based on income level. Age was identified in FY11, and categorized based on the age distribution as 18-30, 31-40, 41-50, 51-64, and 65 years and older. Race/ethnicity was classified as White, Black, Hispanic, other, and unknown as defined in our prior studies using VA data [22]. Co-payment status was identified using the 'means test' variable, which is a financial assessment used to determine if a Veteran qualifies for free healthcare services without copayment based on his/her income level; variable levels include waived, not waived, and unknown.

\subsubsection{Clinical Characteristics: Co-morbid Conditions}

We identified baseline co-morbidities using ICD-9-CM codes [23] documented through VA inpatient and outpatient encounters during FY10 (Online Resource 2) to identify conditions we hypothesized would be associated with CNS polypharmacy or the adverse outcomes of overdose and SRB. The following conditions previously associated with SRB/overdose and commonly treated with
CNS medications are TBI, PTSD, depression, anxiety, substance use disorder (SUD), bipolar disorder, schizophrenia, headache, pain, insomnia, prior SRB, and prior overdose [15]. Due to a statistically significant interaction among TBI, PTSD, and depression with CNS polypharmacy in early analyses, we created a TBI-PTSDdepression status variable to describe the association of each condition or a combination of these conditions with CNS polypharmacy [6]. TBI-PTSD-depression status included the following eight categories: none of these comorbidities; only TBI; only PTSD; only depression; TBI and PTSD; TBI and depression; PTSD and depression; TBI, PTSD and depression [7].

\subsubsection{Adverse Events}

We used ICD-9-CM codes from national VA inpatient and outpatient data to identify alcohol/drug overdose and SRBs (suicidal attempts and ideations) in FY11; we used the same ICD-9-CM codes to identify prior overdose and SRBs in FY0 (Online Resource 2).

\subsection{Statistical Analysis}

We identified IAVs with and without CNS polypharmacy and used the Chi-square test and Haberman's adjusted residual to identify variables that were significantly different and cells that were significantly higher or lower than expected by chance [24]. Next, we determined the prevalence of drug/alcohol overdose and SRB. We used multivariable logistic regression analyses to examine the association of demographic and clinical characteristics with CNS polypharmacy. Similar multivariable logistic regression analyses were used to examine the associations of CNS polypharmacy with overdose and SRB in separate analyses, adjusting for demographic and clinical characteristics. We calculated $99 \%$ confidence intervals (CIs) for odds ratios to correct for the high likelihood of finding statistically significant results due to our large sample size and also tested potential explanatory variables for multicollinearity. While variables with overlapping CIs may be significantly different, we used a conservative approach identifying non-overlapping CIs to determine significantly different associations among variables in each model [25].

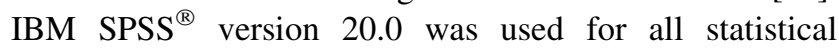
analyses.

\section{Results}

Of 303,716 IAVs who met inclusion criteria, 25,546 $(8.4 \%)$ were dispensed five or more distinct CNS medications. Table 1 shows demographic and clinical 
Table 1 Association of baseline demographic and clinical characteristics with central nervous system (CNS) medication Polypharmacy in Iraq and Afghanistan war Veterans: Bivariate description and results of logistic regression

\begin{tabular}{|c|c|c|c|}
\hline & $\begin{array}{l}\text { CNS polypharmacy } \\
{[n=25,546(8.4 \%)]}\end{array}$ & $\begin{array}{l}\text { No CNS polypharmacy } \\
{[n=278,170(91.6 \%)]}\end{array}$ & AOR $(99 \% \mathrm{CI})$ \\
\hline \multicolumn{4}{|l|}{ Age } \\
\hline Mean (SD), years & $35.91(9.17)$ & $35.92(9.92)$ & $\begin{array}{l}t=-0.82 \\
p=0.41\end{array}$ \\
\hline Median (IQR), years & $33(29-43)$ & $32(28-43)$ & \\
\hline \multicolumn{4}{|l|}{ Groups, years } \\
\hline $17-30$ & $9276(36.3)$ & $114,521(41.2)$ & Reference \\
\hline $31-40$ & $8546(33.5)$ & $76,083(27.4)$ & $1.19(1.14-1.25)$ \\
\hline $41-50$ & $5517(21.6)$ & $59,815(21.5)$ & $1.10(1.04-1.16)$ \\
\hline $51-64$ & $2175(8.5)$ & $26,881(9.7)$ & $1.06(0.98-1.13)$ \\
\hline $65+$ & $32(0.1)$ & $870(0.3)$ & $0.60(0.36-0.95)$ \\
\hline \multicolumn{4}{|l|}{ Gender } \\
\hline Women & $3624(14.2)$ & $36,932(13.3)$ & Reference \\
\hline Men & $21,922(85.8)$ & $241,238(86.7)$ & $0.89(0.84-0.94)$ \\
\hline \multicolumn{4}{|l|}{ Race/ethnicity } \\
\hline White & $17,400(68.1)$ & $174,494(62.7)$ & Reference \\
\hline Black & $3600(14.1)$ & 48,865 (17.6) & $0.69(0.65-0.72)$ \\
\hline Hispanic & $3205(12.5)$ & $32,593(11.7)$ & $0.99(0.94-1.05)$ \\
\hline Other & $817(3.2)$ & $11,416(4.1)$ & $0.79(0.71-0.87)$ \\
\hline Unknown & $524(2.1)$ & $10,802(3.9)$ & $0.66(0.58-0.74)$ \\
\hline \multicolumn{4}{|l|}{ Co-payment status } \\
\hline Exempt from co-payment & $22,755(89.1)$ & $62,580(71.9)$ & Reference \\
\hline Not exempt from co-payment & $2041(8.9)$ & $62,580(22.5)$ & $0.53(0.50-0.57)$ \\
\hline Unclassified & $750(2.9)$ & $15,703(5.6)$ & $1.28(1.15-1.42)$ \\
\hline \multicolumn{4}{|l|}{ Branch of service } \\
\hline Army & $18,640(73.0)$ & $174,602(62.8)$ & Reference \\
\hline Marine corps & $2700(10.6)$ & $37,235(13.4)$ & $0.73(0.69-0.78)$ \\
\hline Air force & $1889(7.4)$ & $29,881(10.7)$ & $0.76(0.71-0.81)$ \\
\hline Navy/coast guard & $2317(9.1)$ & $36,452(13.1)$ & $0.75(0.70-0.80)$ \\
\hline \multicolumn{4}{|l|}{ Co-morbidities } \\
\hline Substance use disorder & $9459(37.0)$ & $38,117(13.7)$ & $2.25(2.16-2.35)$ \\
\hline Bipolar disorder & $4828(18.9)$ & $11,902(4.3)$ & $2.57(2.16-2.35)$ \\
\hline Pain & $20,040(78.4)$ & $126,244(45.4)$ & $2.86(2.73-2.99)$ \\
\hline Headache & $11,358(44.5)$ & $39,775(14.3)$ & $3.08(2.96-3.20)$ \\
\hline Anxiety & $10,563(41.3)$ & $42,832(15.4)$ & $2.32(1.85-2.51)$ \\
\hline Schizophrenia & $570(2.2)$ & $1596(0.6)$ & $2.15(1.85-2.51)$ \\
\hline Insomnia & 10,707 (41.9) & $50,435(18.1)$ & $1.98(1.91-2.06)$ \\
\hline \multicolumn{4}{|l|}{$\mathrm{TBI}+\mathrm{PTSD}+$ depression } \\
\hline No TBI, depression, or PTSD & $1378(5.4)$ & $147,178(52.9)$ & Reference \\
\hline TBI only & $199(0.8)$ & $7161(2.6)$ & $1.83(1.49-2.24)$ \\
\hline PTSD only & $3370(13.2)$ & $36,060(13.0)$ & $6.50(5.96-7.10)$ \\
\hline Depression only & $2599(10.2)$ & $24,206(8.7)$ & $6.42(5.86-7.04)$ \\
\hline $\mathrm{TBI}+\mathrm{PTSD}$ & $2140(8.4)$ & $11,923(4.3)$ & $8.37(7.58-9.24)$ \\
\hline TBI + depression & $347(1.4)$ & $2033(0.7)$ & $7.09(5.95-8.45)$ \\
\hline PTSD + depression & $9593(37.6)$ & $36,738(13.2)$ & $12.98(11.97-14.07)$ \\
\hline TBI + PTSD + depression & $5920(23.2)$ & $12,871(4.6)$ & $15.30(14.00-16.73)$ \\
\hline
\end{tabular}


Table 1 continued

\begin{tabular}{lccc}
\hline & $\begin{array}{l}\text { CNS polypharmacy } \\
{[n=25,546(8.4 \%)]}\end{array}$ & $\begin{array}{l}\text { No CNS polypharmacy } \\
{[n=278,170(91.6 \%)]}\end{array}$ & AOR (99\% CI) \\
\hline $\begin{array}{l}\text { Past adverse event } \\
\text { Drug/alcohol overdose } \\
\text { Suicide attempt }\end{array}$ & $188(0.7 \%)$ & $282(0.1 \%)$ & $1.51(1.11-2.05)$ \\
\hline
\end{tabular}

$A O R$ adjusted odds ratio, $C I$ confidence interval, $I Q R$ interquartile range, $C N S$ central nervous system, $P T S D$ post-traumatic brain disorder, $T B I$ traumatic brain injury

characteristics of patients with and without CNS polypharmacy and the results of logistic regression predicting CNS polypharmacy. Women and individuals between 31 and 50 years of age were more likely to have CNS polypharmacy. Marine Corps veterans were less likely to have CNS polypharmacy than those in other service branches.

\subsection{Characteristics Associated with Central Nervous System (CNS) Polypharmacy}

Logistic regression analyses indicated that individuals diagnosed with headache and other pain conditions were significantly more likely to have CNS polypharmacy than individuals without these diagnosed conditions (Table 1). Individuals with a diagnosis of TBI, PTSD, and/or depression were significantly more likely to have CNS polypharmacy than IAVs without any of these diagnoses. Those diagnosed with PTSD or depression alone or in combination with TBI were more likely to have CNS polypharmacy than those diagnosed with TBI alone. Individuals diagnosed with both PTSD and depression had a significantly higher likelihood of CNS polypharmacy than those diagnosed with only PTSD or depression and the addition of TBI diagnosis to either PTSD or depression diagnosis did not increase the likelihood of CNS polypharmacy, as there was overlap in the CIs. Finally, those previously diagnosed with overdose event and suicide attempt were also significantly more likely to have CNS polypharmacy than those without a diagnosis.

\subsection{Characteristics Associated with Overdose and Suicide-Related Behavior}

Among the cohort, $515(0.2 \%)$ were diagnosed with overdose and $4229(1.4 \%)$ were diagnosed with SRBs in FY11.

After controlling for demographic and clinical characteristics including prior overdose and prior SRB we found that CNS polypharmacy was significantly associated with both overdose and SRB. Individuals with CNS polypharmacy had 3.85 higher odds of overdose (99\% CI
2.95-5.04) than those without CNS polypharmacy, and individuals with CNS polypharmacy had significantly higher odds of SRB (AOR 3.94; $99 \%$ CI 3.58-4.33) than IAVs without CNS polypharmacy.

\section{Discussion}

Polypharmacy and CNS polypharmacy specifically has been examined in older individuals and has been identified as a risk factor for adverse drug events [26, 27]. This has not been examined broadly in younger adults, possibly because they generally receive fewer medications, have adequate hepatic metabolism and renal clearance, and are less sensitive to CNS-acting medications such as opioids, benzodiazepines, and antipsychotics [9, 28-31]. Our findings of relatively high polypharmacy are surprising given the young age of this cohort. However, evaluation of the co-morbidity profiles suggest that multimorbidity may explain the high rates of polypharmacy in IAVs, who have been identified as a patient population with a high prevalence of TBI, PTSD, and pain (also known as the polytrauma clinical triad (PCT) [14, 32, 33]. Recent studies have found that depression and headaches are also highly prevalent and co-morbid with PTSD and TBI in IAVs [34-36]. Pharmacotherapy for these conditions and associated symptoms typically consists of CNSacting medications as recommended by the VA/DoD treatment guidelines [17-20]. Since treatment guidelines are usually aimed at treating specific conditions, multimorbid patients are more likely to receive multiple CNSacting medications, each indicated by a separate guideline. Each medication is appropriate in isolation but in combination CNS drugs may increase the risk for adverse drug effects and events. Management of the chronic conditions examined in this study relies heavily on evidence-based therapies [37]; however, multimorbidity may challenge the determination of a best practice approach. Additional research is needed to better understand how CNS polypharmacy may be the result of interplay between guidelines for co-morbid conditions and problems with coordination of care. 
Recent public attention has focused on improving treatment for IAVs with psychiatric, pain, and SUD, particularly related to reducing risk for substance abuse, medication burden, and drug overdose [38]. Bohnert et al. [39] found accidental overdose to be associated with both psychiatric and SUDs. SUDs, and specifically opioid use disorders, carry the highest risk of accidental overdose. Recent work by Finley et al. [15] demonstrated that Veterans with SUD or combinations of PCT conditions (especially co-morbid PTSD + depression) were more likely to have diagnoses for SRB. However, to our knowledge, this is the first systematic study to examine the association of adverse outcomes among IAVs receiving multiple CNS-acting medications. Our work shows that even after controlling for all other diagnosed co-morbidities, CNS polypharmacy was independently associated with drug/alcohol overdose and SRB. This may be due to pharmacodynamic drug interactions, concomitant use of these medications with other substances such as alcohol, or merely an artifact of treating patients with multimorbidity. If the latter, the significant association may be an indicator of disease severity that can be used clinically to identify atrisk individuals and refer them to interdisciplinary specialty care.

Given the multimorbidity of this population, vigilance and organization of care is vital to avoid harmful drug interactions and to improve patient outcomes [29]. In a study examining poly-sedative prescribing among Veterans over 8 years, $78 \%$ of the dual opioid-benzodiazepine prescriptions were written by separate providers [10], suggesting that suboptimal coordination of care in conjunction with multiple guidelines for care may lead to suboptimal prescribing, including high severity drug interactions and possible unintentional drug overdose [39].

The unprecedented rise of unintentional drug overdose in the US population [5] coupled with our findings related to CNS polypharmacy highlights the need for applicable evidence-based strategies that healthcare providers can use to effectively manage potential risks associated with CNS polypharmacy in Veterans and the general population. Our study uses five or more dispensed CNS medications in assessing risk of adverse events. It is possible that this, or potentially less rigorous criteria, could be used as a guideline for healthcare providers when evaluating patients with multiple CNS-acting medications. Further research is needed to determine if CNS polypharmacy could be used as an effective screening tool to identify patients whose treatment plan may need refinement in order to prevent overdose, SRB, and other adverse events and outcomes.

Our study has several limitations. First, our definition of CNS polypharmacy (five or more prescribed CNS medications) may be considered conservative compared to studies in elderly adults using two or more CNS drugs [16], and Bernardy et al.'s [10] criteria of three or more sedative classes. However, given that nearly $9 \%$ of the cohort met our CNS polypharmacy measure, we believe this is a clinically relevant threshold that may be used to identify individuals at risk of adverse outcomes. Our data did not examine the simultaneous use of medications, dosing, adherence, or whether medications were scheduled or used as needed. More detailed analysis including attention to concomitant drug use, specific co-prescribed medications, and dose is needed to determine if particular classes of medications are more closely associated with these adverse events.

In addition, ICD-9 codes were used to identify suicidal ideation and attempts, which results in a conservative assessment of SRB as data on completed suicides were not available. Information on severity of ideation or the lethality of any suicide attempt was not available. Our analysis also does not take into account severity of associated co-morbidities so we do not know how the severity of a condition affects CNS polypharmacy, overdose, or suicide-related behavior in our cohort. This may be relevant for some conditions such as TBI where studies with more clinically rich data indicate that TBI severity may play a role in suicide risk [9, 40]. Lastly, the study examined associations in cross-sectional data. An examination of the timing of each CNS medication and the adverse outcomes was too complex for this 'signal finding' study; therefore, causality and directionality of identified correlates cannot be determined. However, these findings indicate that a more complex assessment of CNS polypharmacy is needed; these studies would include: different definitions of CNS polypharmacy; determination of whether specific CNS medications are more important than others when considering this problem; and timing between CNS polypharmacy and outcomes.

\section{Conclusions}

The results of this study suggest that IAVs, a relatively young patient population characterized by frequent multimorbidity, are likely to experience CNS polypharmacy. Our analyses further suggest that CNS polypharmacy is independently associated with SRB and overdose, indicating that a more focused evaluation of CNS polypharmacy is needed. These data suggest that CNS polypharmacy may be used as a 'trigger-tool' [41] to identify individuals who may benefit from referral to a tailored interdisciplinary treatment team comprised of experts from the relevant fields (pharmacy, internal medicine, neurology, physical medicine and rehabilitation, rheumatology, psychiatry, and addiction medicine). These teams ideally would work together to optimize medication 
profiles and treatment plans, as well as examine available non-pharmacological treatment options in order to minimize potential adverse events.

Acknowledgments Bill Kazanis, M.S. of South Texas Veterans Healthcare System, San Antonio, Texas, provided limited statistical support for this article. The funding sources had no role in the design and conduct of the study; collection, management, analysis, and interpretation of the data; preparation, review, or approval of the manuscript; and decision to submit the manuscript for publication. The views and opinions expressed in this report are those of the authors and should not be construed to represent the views of the Department of Veterans Affairs or the United States government.

\section{Compliance with Ethical Standards}

Ethical approval This study was approved by the institutional review boards of the University of Texas Health Sciences Center San Antonio and the Edith Nourse Rogers Memorial VA Hospital with a waiver of informed consent in accordance with the ethical standards of the declaration of Helsinki.

Conflict of interest Kangwon Song, Carlos A. Jaramillo, Erin P. Finley, and Mary Jo Pugh are employees of the Department of Veterans Affairs. Carlos A. Jaramillo, Erin P. Finley, and Mary Jo Pugh received grant funding from the Department of Veterans Affairs. Jennifer S. Potter received consulting fees and has provided expert testimony. Garen Collett has no conflicts of interest that are directly relevant to the content of this study.

Funding This study was supported by the Veterans Health Administration Health Services Research and Development Services, DHI 09-237 (Dr. Pugh, principal investigator).

Open Access This article is distributed under the terms of the Creative Commons Attribution-NonCommercial 4.0 International License (http://creativecommons.org/licenses/by-nc/4.0/), which permits any noncommercial use, distribution, and reproduction in any medium, provided you give appropriate credit to the original author(s) and the source, provide a link to the Creative Commons license, and indicate if changes were made.

\section{References}

1. Jeffery DD, May L, Luckey B, Balison BM, Klette KL. Use and abuse of prescribed opioids, central nervous system depressants, and stimulants among U.S. active duty military personnel in FY 2010. Mil Med. 2014;179:1141-8.

2. A review of how prescribed psychiatric medications could be driving members of the armed forces and vets to acts of violence and suicide: a report by citizens commission on human rights international April 2014. 1st ed. Los Angeles: Citizens Commission on Human Rights; 2014:1-34. Available at: http://www. veterans.senate.gov/imo/media/doc/For\%20the\%20Record\%20\%20CCHR\%204.30.14.pdf. Accessed 3 Mar 2015.

3. Frances, AJ. Polypharmacy, PTSD, and accidental death from prescription medication polypharmacy, PTSD, and accidental death from prescription medication. http://www.psychiatrictimes. $\mathrm{com} / \mathrm{ptsd} /$ polypharmacy-ptsd-and-accidental-death-prescriptionmedication. Accessed 31 May 2015.

4. Kelman B, Schmenner D. Marine PTSD: Battling the shock of war. Desert Sun. 2014 http://www.desertsun.com/story/news/ 2014/03/24/marine-ptsd-twentynine-palms-shock-war/6698933/. Accessed 29 Mar 2015.

5. CDC Grand Rounds: Prescription Drug Overdoses-a U.S. Epidemic [Internet]. [cited 2015 Jun 5]. Available from: http://www. cdc.gov/mmwr/preview/mmwrhtml/mm6101a3.htm.

6. Cifu, DX. Traumatic Brain Injury, Posttraumatic stresss disorder, and pain diagnoses in OIF/OEF/ONE Veterans. Available from: http://www.rehab.research.va.gov/jour/2013/509/page1169.html.

7. Jaramillo CA, Cooper DB, Wang C-P, Tate DF, Eapen BC, York GE, et al. Subgroups of US IRAQ and Afghanistan veterans: associations with traumatic brain injury and mental health conditions. Brain Imaging Behav. 2015;9(3):445-455.

8. French DD, Bair MJ, Bass E, Campbell RR, Siddharthan K. Central nervous system and musculoskeletal medication profile of a veteran cohort with blast-related injuries. J Rehabil Res Dev. 2009;46:463-8.

9. Seal KH, Shi Y, Cohen G, Cohen BE, Maguen S, Krebs EE, et al. Association of mental health disorders with prescription opioids and high-risk opioid use in US veterans of Iraq and Afghanistan. JAMA, J Am Med Assoc. 2012;307:940-7.

10. Bernardy NC, Lund BC, Alexander B, Friedman MJ. Increased polysedative use in veterans with posttraumatic stress disorder. Pain Med Malden Mass. 2014;15:1083-90.

11. Tiihonen J, Suokas JT, Suvisaari JM, Haukka J, Korhonen P. Polypharmacy with antipsychotics, antidepressants, or benzodiazepines and mortality in schizophrenia. Arch Gen Psychiatry. 2012;69:476-83.

12. Post-Deployment Health Group. Epidemiology Program PostDeployment Health Group. Report on VA Facility Specific Operation Enduring Freedom (OEF), Operation Iraqi Freedom (OIF), and Operation New Dawn (OND) Veterans Coded with Potential PTSD: Cumulative from 1st quarter FY 2002 through 1st quarter FY 2013 [Internet]. Department of Veterans Affairs; 2015 [cited 2015 Dec 3]. Available from: http://www. publichealth.va.gov/docs/epidemiology/ptsd-report-fy2012-qtr3. pdf.

13. Kane RL. Understanding health care outcomes research. 2nd ed. Sudbury: Jones and Bartlett; 2006.

14. Pugh MJ, Finley EP, Copeland LA, Wang C-P, Noel PH, Amuan $\mathrm{ME}$, et al. Complex comorbidity clusters in OEF/OIF veterans: the polytrauma clinical triad and beyond. Med Care. 2014;52:172-81.

15. Finley EP, Bollinger M, Noël PH, Amuan ME, Copeland LA, Pugh JA, et al. A national cohort study of the association between the polytrauma clinical triad and suicide-related behavior among us veterans who served in Iraq and Afghanistan. Am J Public Health. 2015;105:380-7.

16. Hanlon J, Boudreau R, Roumani Y, Newman A, Ruby C, Wright $R$, et al. Number and dosage of central nervous system medications on recurrent falls in community elders: the health, aging and body composition study. J Gerontol A Biol Sci Med Sci. 2009;64A:492-8.

17. Chou R, Fanciullo GJ, Fine PG, Adler JA, Ballantyne JC, Davies $\mathrm{P}$, et al. Clinical guidelines for the use of chronic opioid therapy in chronic noncancer pain. $\mathrm{J}$ Pain Off $\mathrm{J}$ Am Pain Soc. 2009;10:113-30.

18. Management of Concussion/mTBI Working Group. VA/DoD clinical practice guideline for management of concussion/mild traumatic brain injury. J Rehabil Res Dev. 2009;46:CP1-68.

19. Management of MDD Working Group. Management of Major Depressive Disorder (MDD) (2009)_VA/DoD Clinical Practice Guidelines. 2008 [cited 2015 Dec 4]; Available from: www. healthquality.va.gov/guidelines/MH/mdd/MDDFULL053013. pdf.

20. Management of PTSD Working Group. Management of PostTraumatic Stress Disorder and Acute Stress Reaction (2010)_ 
VA/DoD Clinical Practice Guidelines. 2010 [cited 2015 Dec 4]; Available from: http://www.healthquality.va.gov/ptsd.ptsd_full. pdf.

21. Spelman JF, Hunt SC, Seal KH, Burgo-Black AL. Post deployment care for returning combat veterans. J Gen Intern Med. 2012;27:1200-9.

22. Hope OA, Zeber JE, Kressin NR, Bokhour BG, Vancott AC, Cramer JA, et al. New-onset geriatric epilepsy care: race, setting of diagnosis, and choice of antiepileptic drug. Epilepsia. 2009;50:1085-93.

23. Buck CJ. 2010 ICD-9-CM for hospitals, volumes 1,2 and 3. Professional ed. Maryland Heights: Saunders Elsevier; 2010.

24. Agresti A. Applied categorical data analysis. Technometrics. 1988;30:456.

25. Cumming G, Finch S. Inference by eye: confidence intervals and how to read pictures of data. Am Psychol. 2005;60:170-80.

26. Wright RM, Roumani YF, Boudreau R, Newman AB, Ruby CM, Studenski SA, et al. Effect of central nervous system medication use on decline in cognition in community-dwelling older adults: findings from the health, aging and body composition study. J Am Geriatr Soc. 2009;57:243-50.

27. French DD, Campbell R, Spehar A, Cunningham F, Foulis P. Outpatient medications and hip fractures in the US: a national veterans study. Drugs Aging. 2005;22:877-85.

28. Sotaniemi EA, Arranto AJ, Pelkonen O, Pasanen M. Age and cytochrome P450-linked drug metabolism in humans: an analysis of 226 subjects with equal histopathologic conditions. Clin Pharmacol Ther. 1997;61:331-9.

29. Levine JM. Common drug interactions following traumatic brain injury. J Head Trauma Rehabil. 2013;28:151-4.

30. Cohen BE, Shi Y, Neylan TC, Maguen S, Seal KH. Antipsychotic prescriptions in Iraq and Afghanistan veterans with posttraumatic stress disorder in Department of Veterans Affairs healthcare, 2007-2012. J Clin Psychiatry. 2015;76:406-12.

31. Farinde A. An examination of co-occurring conditions and management of psychotropic medication use in soldiers with traumatic brain injury. J Trauma Nurs. 2014;21:153-7.

32. Lew HL, Otis JD, Tun C, Kerns RD, Clark ME, Cifu DX. Prevalence of chronic pain, posttraumatic stress disorder, and persistent postconcussive symptoms in OIF/OEF veterans: polytrauma clinical triad. J Rehabil Res Dev. 2009;46:697-702.

33. Clark ME, Walker RL, Gironda RJ, Scholten JD. Comparison of pain and emotional symptoms in soldiers with polytrauma: unique aspects of blast exposure. Pain Med Malden Mass. 2009; 10:447-55.

34. Carlson K, Kehle S, Meis L, Greer N, MacDonald R, Rutks I, et al. The assessment and treatment of individuals with history of traumatic brain injury and post-traumatic stress disorder: a systematic review of the evidence [Internet]. Washington (DC): department of veterans affairs (US); 2009 [cited 2013 Oct 28]. Available from: http://www.ncbi.nlm.nih.gov/books/NBK49144/.

35. Poundja J, Fikretoglu D, Brunet A. The co-occurrence of posttraumatic stress disorder symptoms and pain: is depression a mediator? J Trauma Stress. 2006;19:747-51.

36. Shipherd JC, Keyes M, Jovanovic T, Ready DJ, Baltzell D, Worley $\mathrm{V}$, et al. Veterans seeking treatment for posttraumatic stress disorder: what about comorbid chronic pain? J Rehabil Res Dev. 2007;44:153-66.

37. Bosco MA, Gallinati JL, Clark ME. Conceptualizing and treating comorbid chronic pain and PTSD. Pain Res Treat [Internet]. 2013 [cited 2013 Oct 28]; 2013. Available from: http://www.ncbi.nlm. nih.gov/pmc/articles/PMC3684116/.

38. Carey JD Benedict, Frosch D. For some troops, powerful drug cocktails have deadly results. N Y Times [Internet]. 2011 Feb 12 [cited 2015 Jun 5]; Available from: http://www.nytimes.com/ 2011/02/13/us/13drugs.html.

39. Bohnert ASB, Ilgen MA, Ignacio RV, McCarthy JF, Valenstein M, Blow FC. Risk of death from accidental overdose associated with psychiatric and substance use disorders. Am J Psychiatry. 2012;169:64-70.

40. Brenner LA, Ignacio RV, Blow FC. Suicide and traumatic brain injury among individuals seeking veterans health administration services. J. Head Trauma Rehabil. 2011;26:257-64.

41. Handler SM, Hanlon JT. Detecting adverse drug events using a nursing home specific trigger tool. Ann Long Term Care Off $\mathrm{J}$ Am Med Dir Assoc. 2010;18:17-22. 